\title{
Engel, Christine/Menzel, Birgit (Hrsg.): Russland und/als Eurasien. Kulturelle Konfigurationen, 344 S., Frank \& Timme, Berlin 2018. Bassin, Mark/Suslov, Mikhail (Hrsg.): Eurasia 2.0. Russian Geopolitics in the Age of New Media, 374 S., Lexington, Lanham, KY u.a. 2016.
}

\section{Claus Leggewie}

Online publiziert: 9 . September 2020

(C) Der/die Autor(en) 2020

Der australische Kontinent ist eine Insel, die beiden Amerikas, nur durch einen Isthmus verbunden, sind von gewaltigen Ozeanen separiert, Afrika zu Lande nur durch einen schmalen Korridor zu erreichen. Lediglich das räumlich kleine Europa und die gewaltige Landmasse Asiens hängen zusammen, und jeder Versuch, Europa auf den Raum „vom Atlantik bis zum Ural“ (Charles de Gaulle) zu beschneiden, ist ein Konstrukt. Ein Konstrukt wie freilich alle Großraum- und Zivilisationsvorstellungen, wie man nicht zuletzt an Bemühungen erkennen kann, ,Eurasien“ eine geografische Identität, ein kulturelles Substrat oder eine politische Einheit zuzuschreiben. Eben das geschah im Prozess der „Mondialisierung“, die immer neue regionale Entitäten hervorgetrieben hat. Im 19. Jahrhundert haben sich diese Operationen gehäuft und auf Großraumbildung gerichtete Denkschulen und politische Bewegungen hervorgebracht, während sich zum einen Nationalstaaten auf in der Regel begrenzteren Territorien aus größeren Imperien herausgelöst hatten und als Akteure die internationale Politik bestimmten sowie zum anderen neoimperiale Expansionen Platz griffen. Der Geopolitiker Carl Schmitt hat mit seinen im ,Dritten Reich“ entstandenen Publikationen ,Völkerrechtliche Großraumordnung mit Interventionsverbot für raumfremde Mächte. Ein Beitrag zu Reichsbegriff im Völkerrecht“ (1939) und „Land und Meer. Eine weltgeschichtliche Betrachtung“ (1942) die normativ-ideologische Unterscheidung zwischen (angelsächsischen) Seemächten und (europäischen) Landmächten eingeführt.

Verwandt damit sind die im 19. Jahrhundert in Russland aufkommenden, bis heute immer wieder neu aufgelegten und modifizierten neoeurasischen Vorstellungen. In der Putin-Ära haben sie eine gewisse Renaissance erfahren. Vor allem der neurechte Intellektuelle Aleksandr Dugin tut sich mit ihnen hervor und macht sich damit auch in Westeuropa Freunde.

C. Leggewie ( $\triangle)$

Justus-Liebig-Universität Gießen, Gießen, Deutschland

E-Mail: claus.leggewie@zmi.uni-giessen.de 
Eine kulturwissenschaftliche Würdigung des Konzepts Eurasien leistet der von den Slawistinnen Christine Engel (Innsbruck) und Birgit Menzel (Mainz-Germersheim) herausgegebene Sammelband mit deutsch- und englischsprachigen Beiträgen, die auf eine internationale Tagung der Deutschen Gesellschaft für Osteuropakunde im Jahr 2016 zurückgehen. Sie befassen sich mit „Geopolitik und Ideologie“, „Sprache und Kultur“ und „Aspekte(n) von Spiritualität und Identität“, die sich im Eurasien-Konzept finden. Zum einen bieten sie gute Überblicke (Mikhail Suslov, Ilya Kukulin, Sanna Turoma, Wolfgang Eismann, Maria Engström, Sergej Abashin), zum anderen präzise Fallstudien zu Romanen wie Vladimir Sorokins „Tellurija“ (Dirk Uffelmann), Petr Savickijs geografischem Strukturalismus (Tomáš Glanc), zur künstlerischer Produktion von Yerbossyn Meldibekov (Elena Trubina), zur Rezeption des Dschingis Khan in der Sowjetunion und in postsowjetischen Filmen (Katerina Clark, Christine Engel), zur Ausgrabungsstätte Arkaim in der Uralsteppe (Anna Tessmann), zur Identitätskonstruktion des Volks der Chakassen im Süden Sibiriens, zur spirituellen Dimension im Werk des Kybernetikers Vasilij V. Nalimov und zum „Museum der Opfer der politischen Repression“ in Taschkent (Engel). Gerade diese durchweg gut gearbeiteten Studien über wenig bekannte Fälle vermitteln ein Bild der vielseitigen Anschlussfähigkeit eurasischer Identitätskonstrukte, in deren Mittelpunkt aber jeweils die Positionsbestimmung der russischen Vielvölkernation steht und die dabei doch weit über die grobschlächtige politische Instrumentalisierung bei Dugin und anderen hinausgeht. Interessant ist hier auch der Blick ins islamische Zentralasien und nach China. Die Herausgeberinnen leisten damit einen wichtigen Beitrag zur (im Sinne der New Spatial beziehungsweise Entangled Histories) methodischen Neubestimmung und geografischen Ausweitung der Osteuropaforschung.

Diese Absicht verfolgt auch der Sammelband, der von den renommierten Eurasien-Experten Mikhail Suslov (Uppsala) und Mark Bassin (Stockholm) herausgegeben und von Pionierinnen wie Marlene Laruelle (Washington) beliefert wurde. In fünf Sektionen und 15 substanziell-kritischen Beiträgen werden die geopolitischen Ambitionen des heutigen Russland in populären Memes (Saara Ratilainen, Birgit Beumers, Galina Zvereva), explizit politischen Ideologien (Andrei Tsygankov, Marlene Laruelle, Sirke Mäkinen), russischen Großmachtkonzepten (Hanna Smith, Fabian Linde, Per-Arne Bodin), russophilen Medienstrategien (Vlad Strukov, Ryhor Nizhnikau) und im ukrainischen Kontext (Dirk Uffelmann, Greg Simons, Mikhail Suslov) sehr klar herausgearbeitet. Das besondere Augenmerk liegt auf „Eurasia 2.0“, der Popularisierung und Radikalisierung von ohnehin grobschlächtig-militanten EurasienKonzepten in der digitalen Sphäre, wo sie zu kaum steuerbaren Propagandaschlachten ausarten und für systematische Desinformation eingesetzt werden. Hier entsteht, nicht zuletzt unter habituellen Nutzern sozialer Medien, ein virtuelles Eurasien, das Regionalkonflikte befördert und Territorialstreitigkeiten anheizt, der Herrschaftssicherung autokratischer Regime und Parastaaten wie im Donbass dient und auf tückische Weise naive oder auch absichtsvolle Vasallen der russischen Großmachtpolitik im Westen rekrutiert. Laruelle zeigt am Beispiel des kasachischen Aktivisten Geidar Dzhemal die Anschlussfähigkeit des okkulten Faschismus an den Islamismus. In beiden Bänden nur am Rande behandelt wird die wirtschaftspolitische Ausprägung des Eurasien-Konzepts in supranationalen Verbünden, die realwirtschaftlich ohnehin 
kaum eine Bedeutung haben. Eurasien ist im Kern ein russozentrisches Propagandainstrument des ,Russkii Mir“.

Beide Sammelbände sind von großem Wert für das Verständnis der geistig-kulturellen und politischen Lage in Russland und im „eurasischen Raum“. Dessen Konstruiertheit wird im ersten Band sehr deutlich, aber bei dieser kulturwissenschaftlichen Verflüssigung kann man es nicht belassen, wenn man im zweiten Band die machtpolitische Instrumentalisierung von Raumvorstellungen zur Kenntnis nehmen muss, die ohne geografisches und historisches Substrat auskommen und wie bei Dugin und Konsorten in esoterisch-martialischen Fake übergehen. Angesichts dieses Wildwuchses, der wohl auch die Erfinder der nach 1945 zu Recht diskreditierten Geopolitik wie Friedrich Ratzel, Rudolf Kjellén, Halford Mackinder und andere protestieren lassen würde, machen sich die Analysen um die Erarbeitung einer kritischen Geopolitik verdient. Ob sie damit, wie Suslov und Bassin hoffen, Einfluss auf die russische Debatte nehmen können, ist fraglich.

Funding Open Access funding provided by Projekt DEAL.

Open Access Dieser Artikel wird unter der Creative Commons Namensnennung 4.0 International Lizenz veröffentlicht, welche die Nutzung, Vervielfältigung, Bearbeitung, Verbreitung und Wiedergabe in jeglichem Medium und Format erlaubt, sofern Sie den/die ursprünglichen Autor(en) und die Quelle ordnungsgemäß nennen, einen Link zur Creative Commons Lizenz beifügen und angeben, ob Änderungen vorgenommen wurden.

Die in diesem Artikel enthaltenen Bilder und sonstiges Drittmaterial unterliegen ebenfalls der genannten Creative Commons Lizenz, sofern sich aus der Abbildungslegende nichts anderes ergibt. Sofern das betreffende Material nicht unter der genannten Creative Commons Lizenz steht und die betreffende Handlung nicht nach gesetzlichen Vorschriften erlaubt ist, ist für die oben aufgeführten Weiterverwendungen des Materials die Einwilligung des jeweiligen Rechteinhabers einzuholen.

Weitere Details zur Lizenz entnehmen Sie bitte der Lizenzinformation auf http://creativecommons.org/ licenses/by/4.0/deed.de. 